\title{
Sesamol loaded solid lipid nanoparticles: a promising intervention for control of carbon tetrachloride induced hepatotoxicity
}

\author{
Neha Singh ${ }^{1}$, Neeraj Khullar ${ }^{1}$, Vandita Kakkar ${ }^{2}$ and Indu Pal Kaur ${ }^{2 *}$
}

\begin{abstract}
Background: Sesamol, a component of sesame seed oil, exhibited significant antioxidant activity in a battery of in vitro and ex vivo tests including lipid peroxidation induced in rat liver homogenates. Latter established its potential for hepatoprotection. However, limited oral bioavailability, fast elimination (as conjugates) and tendency towards gastric irritation/toxicity (especially forestomach of rodents) may limit its usefulness. Presently, we packaged sesamol into solid lipid nanoparticles (S-SLNs) to enhance its biopharmaceutical performance and compared the efficacy with that of free sesamol and silymarin, a well established hepatoprotectant, against carbon tetrachloride induced hepatic injury in rats, post induction. A self recovery group in which no treatment was given was used to observe the self-healing capacity of liver.
\end{abstract}

Methods: S-SLNs prepared by microemulsification method were administered to rats post-treatment with $\mathrm{CCl}_{4}$ $(1 \mathrm{ml} / \mathrm{kg}$ body weight (BW) twice weekly for 2 weeks, followed by $1.5 \mathrm{ml} / \mathrm{kg}$ BW twice weekly for the subsequent 2 weeks). Liver damage and recovery on treatment was assessed in terms of histopathology, serum injury markers (alanine aminotransferase, aspartate aminotransferase), oxidative stress markers (lipid peroxidation, superoxide dismutase, and reduced glutathione) and a pro-inflammatory response marker (tumor necrosis factor alpha).

Result: S-SLNs $(120.30 \mathrm{~nm})$ at a dose of $8 \mathrm{mg} / \mathrm{kg}$ BW showed significantly better hepatoprotection than corresponding dose of free sesamol (FS; $\mathrm{p}<0.001)$. Effects achieved with S-SLNs were comparable with silymarin (SILY), administered at a dose of $25 \mathrm{mg} / \mathrm{kg}$ BW. Self recovery group confirmed absence of regenerative capacity of hepatic tissue, post injury.

Conclusion: Use of lipidic nanocarrier system for sesamol improved its efficiency to control hepatic injury. Enhanced effect is probably due to: a) improved oral bioavailability, b) controlled and prolonged effect of entrapped sesamol and iii) reduction in irritation and toxicity, if any, upon oral administration. S-SLNs may be considered as a therapeutic option for hepatic ailments as effectiveness post induction of liver injury, is demonstrated presently.

Keywords: Sesamol, SLNs, $\mathrm{CCl}_{4}$, Oxidative stress, Hepatoprotective

\section{Background}

Several of the drugs recommended for the treatment of liver diseases tend to show adverse effects on the liver function [1]. As the current available choice of useful drug treatments for hepatic dysfunction is highly limited [1], hence there is a continuous search for safe, efficient and alternative therapeutic options [2]. Oxidative stress,

\footnotetext{
* Correspondence: indupalkaur@yahoo.com

${ }^{2}$ Department of Pharmaceutics, University Institute of Pharmaceutical

Sciences, Panjab University, Chandigarh 160014, India

Full list of author information is available at the end of the article
}

or production of reactive oxygen species (ROS) is known to initiate and regulate the progression of liver disease, independent of the etiologic agent [3]. Thus, phytochemicals with significant antioxidant activity are being explored extensively for the treatment of liver disease.

Carbon tetrachloride $\left(\mathrm{CCl}_{4}\right)$ induced liver injury is the best-characterized animal model of xenobiotic-induced free radical-mediated hepatotoxicity [4]. $\mathrm{CCl}_{4}$ metabolism begins with the formation of trichloromethyl free radical, $\mathrm{CCl}_{3}{ }^{*}$ through the action of the mixed function cytochrome P450 oxygenase system of the endoplasmic 
reticulum $[4,5]$ which elicits production of reactive oxygen intermediates and causes lipid peroxidation (LPO). Latter is responsible for hepatocellular damage and enhanced production of connective tissue [6,7]. $\mathrm{CCl}_{3}$ * binds to cellular molecules (nucleic acid, protein, lipid), impairing crucial cellular processes such as lipid metabolism, and resulting in fatty degeneration i.e. steatosis [8]. It can also react with oxygen to form the trichloromethyl peroxy radical $\mathrm{CCl}_{3} \mathrm{OO}$, a highly reactive species which initiates the chain reaction of LPO as it attacks and destroys polyunsaturated fatty acids, in particular those associated with phospholipids [8].

Sesamol is a phenol component of sesame oil [9] and is formed by hydrolysis of sesamolin during thermal oxidation [10]. Sesamol, 5-hydroxy-1, 3-benzodioxole or 3,4-methylenedioxyphenol is a well-established antioxidant molecule [11-13]. Its antioxidant activity is attributed to the presence of the benzodioxole group in its ring, which scavenges hydroxyl radical to produce another antioxidant molecule 1,2-dihydroxybenzene [14] that assigns significant hepatoprotective effects to sesamol. Oral bioavailability (BA) of $35.5 \pm 8.5 \%$ has been reported for sesamol in Sprague Dawley rats [15]. Further, sesamol conjugated metabolites were found to be widely distributed in rat tissues, with the highest concentrations in the liver and kidneys upon oral administration of $100 \mathrm{mg} / \mathrm{kg}$ BW in rats, with their rapid elimination via urine and feces within 0-4 h [16]. The Carcinogenic Potency Database indicates that sesamol could be a potential cause of forestomach cancers in rodents with determined $\mathrm{TD}_{50}$ values of 1.35 and $4.5 \mathrm{~g} / \mathrm{kg} /$ day dose in mice and rats respectively [17]. Although the point is not of much concern considering that there are no forestomachs in humans and the $\mathrm{TD}_{50}$ dose is much higher than the pharmacological per oral dose of $50-100 \mathrm{mg} /$ $\mathrm{kg} /$ day reported thus far, however, the observed effect may however be attributed to its irritant nature due to the phenolic group present in its structure [18].

Sesamol has been reported to possess hepatoprotective properties [19-26] in several models of hepatotoxicity. It may however be noted that sesamol till date has been evaluated in acute model of hepatotoxicity carried for a single dose administration, except for a study wherein sesamol was repetitively administered subcutaneously at a dose of $10 \mathrm{mg} / \mathrm{kg}$, at $0,6,12,18,24,30,36$, and $42 \mathrm{~h}$ after cecal ligation and puncture, in rats [19]. Sesamol was invariably administered parenterally through subcutaneous route $[19,20,23]$ or intra peritoneal (i.p) route $[21,22]$ in these studies. Further antioxidant effects in terms of reduced glutathione (GSH), alanine aminotransferase (ALT), aspartate aminotransferase (AST) and LPO were observed mainly at doses $\geq 10 \mathrm{mg}$ e.g. $10 \mathrm{mg}$ [19,22], $20 \mathrm{mg}$ [20] and $41 \mathrm{mg}$ [21] respectively. In an early report the protective effects of sesamol and its related compounds on $\mathrm{CCl}_{4}$ induced liver injury in rats was studied [26]. Sesamol exhibited significant effect in case of i.p, subcutaneous and oral administration by alleviating ALT, AST, lactate dehydrogenase, alkaline phosphatase, direct bilirubin and total bilirubin parameters. However, the study was an acute study wherein $\mathrm{CCl}_{4}$ was administered at a dose of $0.3 \mathrm{ml} / \mathrm{kg}$ and treatment with sesamol was given $2 \mathrm{~h}$ prior or after. Even at a dose as high as $276.24 \mathrm{mg}$ given orally complete restoration of the elevated parameters was not achieved. Previously we have reported that sesamol could be used for preventive therapy for $\mathrm{CCl}_{4}$ induced sub-chronic hepatotoxicity when loaded into a suitable delivery system at a dose of $8 \mathrm{mg} / \mathrm{kg}$ [27].

In view of fast elimination, low $\mathrm{BA}$, an irritant nature and absence of extensive sub-chronic/chronic studies evaluating hepatoprotective effects of sesamol, it was presently proposed to explore the therapeutic and hepatoprotective potential of sesamol loaded into solid lipid nanoparticles (SLNs). It is noteworthy that the present study evaluated the ability of bioavailable sesamol (SSLNs) to provide hepatoprotection post induction of liver injury. This is in sharp contrast to the proposed protective role of these natural agents upon pretreatment. SLNs are an excellent colloidal carrier system $[28,29]$ whose small size $(<200 \mathrm{~nm})$ helps to overwhelm the reticuloendothelial system (RES) pick up such that these particles recirculate or pass several times through liver tissues thus achieving an enhanced (both intensity and duration) effect of the entrapped agent $[27,30]$.

A well established hepatoprotective agent silymarin (SILY) [31] was used to compare the efficacy of S-SLNs and free sesamol (FS). Additionally, a self recovery (SR) group which did not undergo any treatment after induction of hepatotoxicity was also included in the study to rule out false implications of the therapy. The objectives of the present study were to evaluate the efficacy of S-SLNs in terms of serum biochemical markers of liver injury i.e. ALT, AST, antioxidant markers, a pro-inflammatory marker i.e. tumor necrosis factor- $\alpha$ (TNF- $\alpha$ ) and histopathological investigations, in $\mathrm{CCl}_{4}$ induced liver injury in rats. However, only the basic histological, biochemical and molecular studies (due to limitation of animals to be used and funds available) were selected as evaluation parameters because the main objective of the study was not to elicit the mechanism of action of sesamol which is already well illustrated, but to establish the hepatoprotective superiority of the formulated S-SLNs over the free sesamol form- (i) upon oral administration, (ii) at clinically relevant doses, and (iii) as therapeutic agents for chronic liver diseases.

\section{Methods \\ Materials}

Sesamol was obtained as a free gift from Jubilant Life Sciences (Noida, Uttar Pradesh, India); Compritol 888 ATO $^{\circ}$ 
was a gift sample from Gattefosse, USA. Soy Lecithin (Hi Media, India); Tween 80 (S.D. Fine Chemicals, India) and $\mathrm{CCl}_{4}$ (Merck) were also used in the study. All other chemicals and reagents were of analytical grade.

\section{Preparation of S-SLNs}

SLNs were prepared by the microemulsification method, as reported in our previous work [27]. Briefly, polysorbate $80(45.45 \%)$, soy lecithin $(0.58 \%)$ and water were placed together in a beaker and heated to the lipid melting temperature. Lipid $(7.27 \%)$ was also melted at $82-85^{\circ} \mathrm{C}$ separately. Sesamol was added to the aqueous phase containing polysorbate 80 , following which the hot aqueous emulsifier mix, was dropped at once into the lipid melt, under magnetic stirring to obtain a clear microemulsion. The hot microemulsion thus formed, was transferred into an equivalent amount of cold water $\left(\sim 2^{\circ} \mathrm{C}\right)$ under continuous mechanical stirring $(5000 \mathrm{rpm})$ for $1.5 \mathrm{~h}$. In the aqueous medium, SLNs are formed by crystallization of the hot lipid droplets present in the microemulsions [32]. The prepared aqueous SLN dispersion was stored in a refrigerator until further analysis.

\section{Characterization of SLNs}

The average particle size, polydispersity index (PDI) and zeta potential of SLN dispersion was determined using DelsaNano C, Beckman Coulter, Inc. The morphology of SLNs was examined using an electronic transmission microscope (Hitachi H-100, Japan). The total drug content (TDC) and the \% entrapment of sesamol in SLNs were determined as described in our previous work $[27,33]$. Briefly, TDC was estimated spectrophotometrically at $\lambda_{\max }$ of $294 \mathrm{~nm}$ by disrupting $1 \mathrm{~mL}$ of the SLN dispersion using an appropriate volume of chloroform: methanol $(1: 1, \mathrm{v} / \mathrm{v})$. The $\%$ entrapment was estimated by dialysis bag method $[27,33]$ where $1 \mathrm{ml}$ of S-SLN dispersion was dialysed against $100 \mathrm{ml}$ of water to remove the free sesamol, maintained at $37^{\circ} \mathrm{C}$ and stirred at $150 \mathrm{rpm}$, using dialysis bag (12KDa, Hi Media, India). After $15 \mathrm{~min}$ SLN dispersion remaining in the dialysis bag, i.e. the dialysate was disrupted with a suitable quantity of chloroform: methanol $(1: 1, v / v)$ and the clear solution was analysed spectrophotometrically to give a direct measure of the entrapped sesamol/ml of the SLN dispersion.

\section{Animal model and experimental protocol}

Male Wistar rats weighing 150 to 200 gm were used in the study. The experimental protocols were approved by the Institutional Animals Ethical Committee of Panjab University, Chandigarh (letter no 5381/VCD 6/12/10, meeting held on 29.11.10). Rats were fed standard chow diet and tap water ad libitum. The animals were housed 4 to 5 per cage.
Animals were randomly divided into different groups, each having 6 animals except for the naive control (NC) group and per se (PS) groups which comprised of 3 animals each. In the positive control $\left(\mathrm{CCl}_{4}\right)$ group, hepatic injury was induced by oral administration of $1 \mathrm{ml} / \mathrm{kg}$

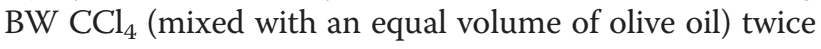
a week for first two weeks followed by $1.5 \mathrm{ml} / \mathrm{kg}$ body weight (BW) for the subsequent two weeks. In the vehicle control (VC) group $1 \mathrm{ml} / \mathrm{kg}$ BW olive oil was administered twice a week for first two weeks and $1.5 \mathrm{ml} / \mathrm{kg}$ for the subsequent two weeks. In the S-SLN group three days after administering the last dose of $\mathrm{CCl}_{4}$ as in the positive control group, S-SLNs were administered at a dose of $8 \mathrm{mg} / \mathrm{kg}$ BW daily for four weeks. Similarly, in the FS group, three days post last dose of $\mathrm{CCl}_{4}$, $\mathrm{FS}$ dissolved in distilled water was administered orally at a dose of $8 \mathrm{mg} / \mathrm{kg}$ BW daily for four weeks. In SILY group three days post last dose of $\mathrm{CCl}_{4}$, SILY (dispersed in $0.7 \% \mathrm{w} / \mathrm{v}$ carboxy methyl cellulose in water) was administered at a dose of $25 \mathrm{mg} / \mathrm{kg} \mathrm{BW}$ [34] daily for four weeks. In the SR group after the final dose of $\mathrm{CCl}_{4}$ was administered, rats were left for self recovery for four weeks. In the NC group the animals were kept on normal diet throughout the eight week period of study. In the S-SLNs PS group after four weeks of normal diet SSLNs were administered to rats at a dose equivalent of $8 \mathrm{mg}$ of sesamol/kg BW daily for four weeks. Similarly, in the FS PS, after four weeks of normal diet, FS dissolved in distilled water was administered orally at a dose of $8 \mathrm{mg} / \mathrm{kg}$ BW daily for four weeks. In blankSLNs per se group (Bla-SLNs PS) blank SLNs were administered orally daily for four weeks after rats were kept at normal diet for four weeks. In the $\mathrm{CCl}_{4}$ group animals were sacrificed three days post final dose of $\mathrm{CCl}_{4}$ i.e. on $32^{\text {nd }}$ day and all the other animals were sacrificed on the 60th day, i.e. a day after the final treatment schedule (no treatment in the case of $\mathrm{NC}, \mathrm{VC}$ and SR groups).

Blood sampling was done via retro-orbital plexus, in ether anesthetized rats. Serum was isolated by centrifuging blood samples at $4^{\circ} \mathrm{C}$ for $20 \mathrm{~min}$ at $4000 \mathrm{rpm}$ and was stored at $-20^{\circ} \mathrm{C}$. Rats were sacrificed by cervical dislocation and their livers were harvested after the end of each study. A part of the liver was stored at $10 \%$ buffered formalin for histopathological examinations and the remaining part was homogenized with $10 \%(\mathrm{w} / \mathrm{v})$ cold phosphate -buffered saline (PBS $0.1 \mathrm{Mol} / \mathrm{l}, \mathrm{pH}$ 7.4). The oxidative stress parameters were determined in the liver post -mitochondrial supernatant (PMS) prepared by centrifuging rat liver homogenates in the chilled phosphate buffer, $\mathrm{pH} 7.4$ at 10,500 g for $20 \mathrm{~min}$ at $4{ }^{\circ} \mathrm{C}$. The following tests were performed to assess the therapeutic potential of S-SLNs and other groups under study. 


\section{Histological examinations of liver}

Representative liver tissue of different groups was processed, stained with haematoxylin \& eosin (HE) and examined under the light microscope for steatosis, inflammation, necrosis, fibrosis and cirrhosis. The histological changes were observed on HE stained sections $[27,35]$. Changes in the histological parameters for liver tissue, i.e. fatty changes, inflammation and fibrosis were graded as follows: $(-)$ showing no occurrence and,,++++++ and ++++ as mild, medium, high and severe occurrences respectively.

\section{Estimation of serum liver injury markers}

Serum of animals of the respective groups stored at $20^{\circ} \mathrm{C}$ was used to measure ALT and AST levels according to Reitman \& Frankel's method [36]. For ALT estimation $0.5 \mathrm{ml}$ of the substrate comprising $0.2 \mathrm{M}$ alanine, $2.0 \mathrm{mM}$ ketoglutarate and $100 \mathrm{mM}$ phosphate buffer, $\mathrm{pH}$ 7.4, was taken. However, the substrate for AST comprised of $0.2 \mathrm{M}$ aspartate, $2.0 \mathrm{mM}$ ketoglutarate and $100 \mathrm{mM}$ phosphate buffer, pH 7.4. Both serum AST \& ALT was expressed as nmoles/nL and was compared with sodium pyruvate as the standard.

\section{Estimation of antioxidant parameters}

The oxidative stress parameters were determined in liver PMS.

\section{Estimation of LPO}

The quantitative measurement of LPO in liver was done according to the method of Wills [37]. The malondialdehyde (MDA) content, a measure of LPO, was assayed as liver thiobarbituric acid reactive substances. The results were expressed as nanomoles of MDA per milligram of protein, using the molar extinction coefficient of the chromophore as $1.56 \times 105 \mathrm{M}^{-1} \mathrm{~cm}^{-1}$.

\section{Measurement of superoxide dismutase (SOD)}

SOD activity was assayed according to the method of Kono et al. [38]. SOD activity was expressed in terms of units of SOD per milligram of protein (SOD units/mg Pr).

\section{Estimation of GSH levels}

GSH was estimated by the method of Jollow et al. [39]. The results were expressed as nmoles of GSH per $\mu \mathrm{g}$ of

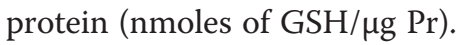

\section{Estimation of pro-inflammatory cytokine, TNF- $a$}

TNF- $\alpha$ levels were measured in liver homogenates using an ELISA kit (RayBiotech, Inc). The assay was performed according to the manufacturer protocol.

\section{Statistical Analysis}

Data expressed as mean \pm standard deviation (SD), were analysed through one way analysis of variance (ANOVA), followed by the Tukey test for comparison of various treatments using Sigma stat 3.5. Differences were considered statistically significant at $\mathrm{p} \leq 0.001$ or $\mathrm{p}<0.05$ as indicated suitably.

\section{Results}

\section{Characterization of SLNs}

The average particle size, PDI and zeta potential of SSLNs was found to be $120.30 \mathrm{~nm}, 0.111$ and $-51.31 \mathrm{mV}$ respectively. When observed under TEM, S-SLNs were found to be spherical in shape (Figure 1). The TDC and \% entrapment of S-SLNs were estimated to be $3.31 \pm$ $0.01 \mathrm{mg} / \mathrm{ml}$ and $73.92 \pm 2.49 \%$ respectively $(\mathrm{n}=3)$.

\section{Histological analysis}

The hepatoprotective effects of S-SLNs were evaluated by histological analysis. Representative views of liver sections are shown in Figure 2 (a-f). Figure 2a represents the VC liver sections stained with $\mathrm{HE}$ showing normal histoarchitecture. The PS and NC groups showed features similar to VC group and hence are not shown. However, liver tissue in the rats treated with $\mathrm{CCl}_{4}$ revealed extensive liver injuries, characterized by fatty cells, inflammatory bridges and vascular bridges (Figure 2b). Howsoever, treatment with S-SLNs (Figure 2c) improved the state of steatosis, ameliorated inflammation as compared to $\mathrm{CCl}_{4}$ group. In comparison, $\mathrm{FS}$ treatment did not restore the histoarchitecture as indicated by the presence of steatosis, and dense portal inflammation (Figure 2d). SILY group, also showed a significant improvement, with medium steatosis and portal inflammation being evident in

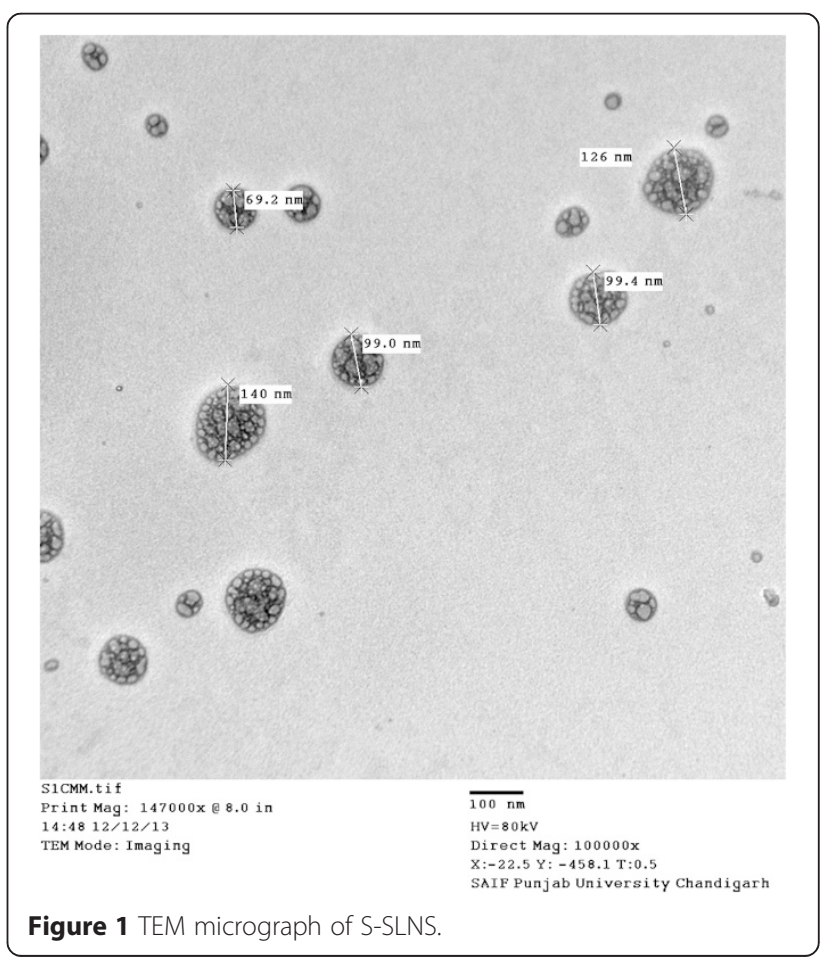



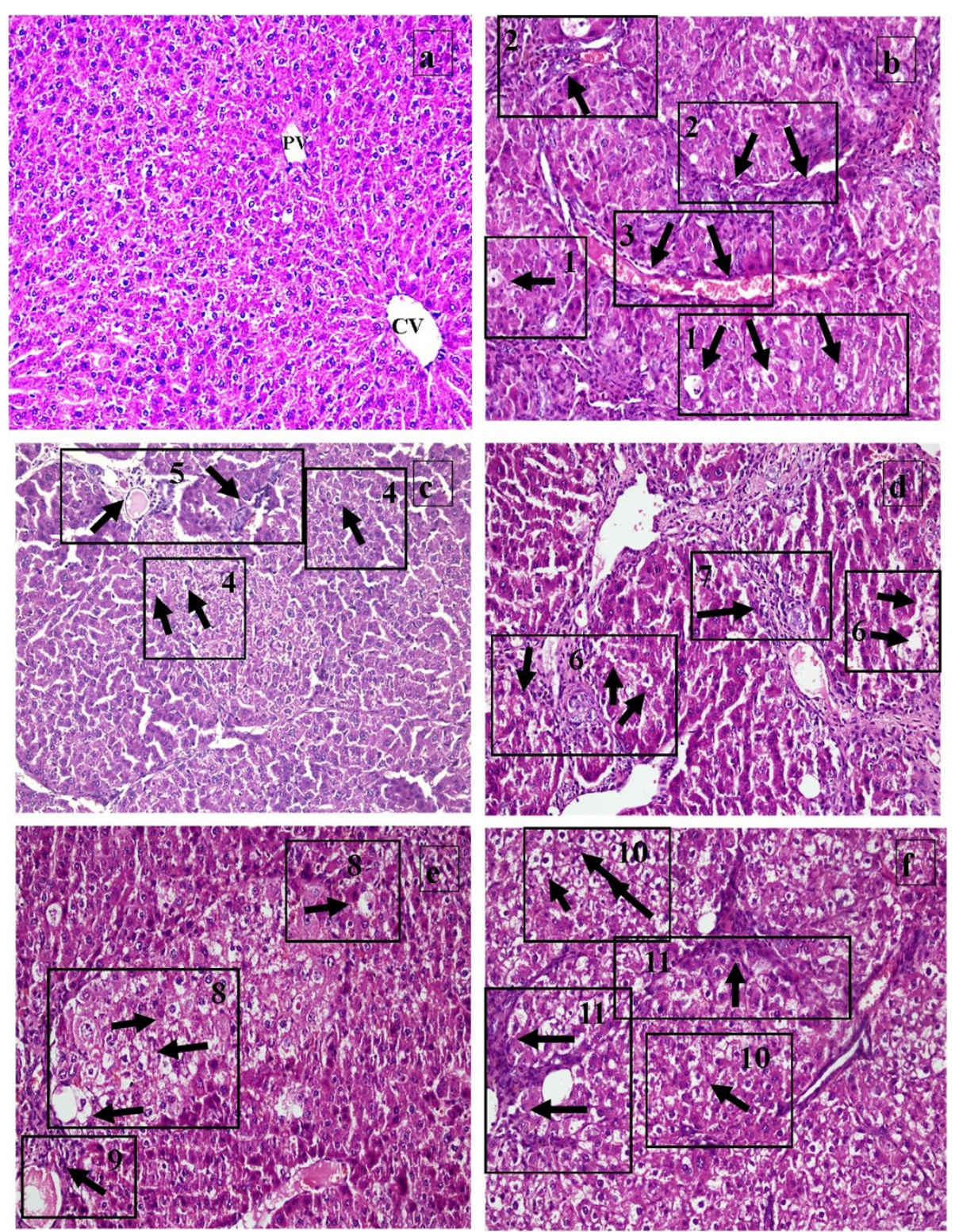

Figure 2 Haemotoxylin and eosin (HE) stained liver section of vehicle control animals showing polyhedral hepatocytes (H), central vein (CV) and portal vein (PV) (2a); rats administered $\mathrm{CCl}_{4}$ showing altered histoarchitecture characterized by fatty changes i.e. steatosis [1], inflammatory bridges [2] and vascular bridges [3] 2(b); S-SLNs treated rats showing mild steatosis [4] and medium portal inflammation [5] 2(c); FS treated rats showing medium steatosis [6], high inflammation [7]; SILY treated rats showing medium steatosis [8] and inflammation [9] 2(e); and untreated SR group showing altered histoarchitecture with high occurrence of steatosis [10] and inflammation [11] 2(f). Representative views of each group are presented at 200X magnification.

the observed histological sections (Figure 2e) which were comparable to the S-SLNs group. The SR group showed (Figure 2f) altered histoarchitecture with high occurrence of steatosis and inflammation indicating that the liver is unable to restore its architecture in the absence of any treatment. The obtained results are in terms of the extent of hepatic injury and compiled in Table 1.

\section{Serum liver injury markers}

Administration of $\mathrm{CCl}_{4}$ led to $2570.07 \%$ increase in the ALT levels as compared to the VC group.
However, S-SLNs and FS treatment significantly decreased the elevated ALT levels by $52.59 \pm 2.37 \%$ and $34.12 \pm 3.69 \%$ respectively. Results obtained with $\mathrm{S}$ SLNs were significantly better $(\mathrm{p}<0.001)$ than $\mathrm{CCl}_{4}$, FS and SR groups. The effect was, however comparable to SILY (as shown in Table 2), though the latter was administered at a 3 times higher dose of $25 \mathrm{mg} / \mathrm{kg}$ BW. Similarly, AST levels were increased by $890.89 \%$ in the $\mathrm{CCl}_{4}$ group with respect to the $\mathrm{VC}$ group and the level significantly decreased in S-SLNs $(\mathrm{p}<0.001)$ treated group as compared to the $\mathrm{CCl}_{4}, \mathrm{FS}$ and $\mathrm{SR}$ 
Table 1 Histological grading of $\mathrm{CCl}_{4}$ induced hepatic injury in male Wistar rats

\begin{tabular}{llll}
\hline Group & Fatty changes & Inflammation & Fibrosis \\
\hline NC & - & - & - \\
VC & - & - & - \\
S-SLNs PS & - & - & - \\
FS PS & - & - & - \\
Bla SLNs PS & - & - & - \\
CCl & & ++++ & ++++ \\
S-SLNs & ++++ & ++ & ++ \\
FS & + & +++ & +++ \\
SILY & ++ & ++ & ++ \\
SR & ++ & +++ & ++++ \\
\hline -, normal occurrence; +, mild occurrence; ++, medium occurrence; +++, high \\
occurrence; ++++, severe occurrence.
\end{tabular}

groups. S-SLNs attenuated the increased AST levels by $75.68 \pm 3.61 \%$. The latter may be expressed as a 4.13 times improvement as compared to a 2.09 times improvement observed in the FS group.

\section{Antioxidant parameters}

LPO

LPO expressed as MDA levels increased significantly $(\mathrm{p}<$ 0.001) in $\mathrm{CCl}_{4}$ group as compared to $\mathrm{NC}$ and $\mathrm{VC}$ group. There was attenuation in LPO as indicated by a significant decrease in levels of MDA in S-SLNs $(\mathrm{p}<0.001)$ group with respect to $\mathrm{CCl}_{4}$ group. S-SLNs treatment was significantly more effective $(2.38$ fold $)$ in reducing $(\mathrm{p}<0.001)$ MDA levels as compared to SR (1.64 fold) group and FS (1.96 fold; $\mathrm{p}<0.05)$ group. The attenuation was comparable to SILY group (2.04 fold). There was no significant difference in the values of $\mathrm{NC}, \mathrm{VC}$ and PS groups (Figure 3).

\section{SOD levels}

SOD levels were found to be significantly $(\mathrm{p}<0.001)$ low in $\mathrm{CCl}_{4}$ group as compared to $\mathrm{NC}$ and $\mathrm{VC}$ group.
Treatment with S-SLNs however, increased the SOD levels $(4.19$ times) significantly $(\mathrm{p}<0.05)$ as compared to FS (2.91 times) and SILY (3.01 times) groups. The SR group showed 2.56 times improvement in SOD levels (Figure 4), however the effects achieved in the S-SLNs were significantly better $(p=0.001)$ than SR group. There was no significant difference in the values of $\mathrm{NC}$, VC and PS groups.

\section{GSH levels}

The level of total GSH was significantly reduced ( $\mathrm{p}<$ 0.001 ) by $\mathrm{CCl}_{4}$ as compared to the $\mathrm{VC}$ and $\mathrm{NC}$ group. Treatment with S-SLNs countered the inhibitory effect of $\mathrm{CCl}_{4}$ by significantly $(\mathrm{p}<0.001)$ increasing $(6.49$ times) the GSH levels as compared to FS (4.81 times), SILY (4.94 times) and SR (4.44 times) groups. Furthermore, Bla-SLNs PS and S-SLNs PS groups very interestingly showed a significant increase in the GSH levels in comparison to $\mathrm{NC}$ and VC groups (Figure 5).

\section{Inflammatory response}

The levels of the proinflammatory cytokine TNF- $\alpha$ were determined in the liver. As shown in Figure 6, compared with the VC group, level of TNF- $\alpha$ was found to be significantly elevated in the $\mathrm{CCl}_{4}(1379.76 \pm 128.85 \%)$ group. S-SLN treatment significantly reduced $(2.47$ times) the elevated levels of TNF- $\alpha(\mathrm{p}<0.001)$ as compared to FS (1.91 times) and SR groups (1.62 times). However, SILY group showed vast improvement (4.34 times) which was higher than all other treatment groups. There was no significant difference in the values of $\mathrm{NC}$, $\mathrm{VC}$ and PS groups.

\section{Discussion}

The present investigation was an endeavor to explore hepatoprotective effects of S-SLNs in comparison to free drug and silymarin and establish S-SLNs as a therapeutic option for hepatic damage post its induction. Scarce data [40] including couple from our laboratory $[27,30]$ on the

Table $2 \%$ inhibition in ALT and AST levels with respect to $\mathrm{CCl}_{4}$ group, \% increase in ALT and AST levels with respect to VC group and alleviation of the hepatic damage in terms of fold reduction in increased enzyme levels change with respect to $\mathrm{CCl}_{4}$

\begin{tabular}{|c|c|c|c|c|c|}
\hline \multirow[b]{2}{*}{ Groups } & \multirow[b]{2}{*}{ Dose $(\mathrm{mg} / \mathrm{kg})$} & \multicolumn{2}{|l|}{ ALT Levels } & \multicolumn{2}{|l|}{ AST Levels } \\
\hline & & $\begin{array}{l}\% \text { Inhibition versus } \mathrm{CCl}_{4} \text { group } \\
\text { (\% increase with respect to VC) }\end{array}$ & $\begin{array}{l}\text { Alleviation (Fold } \\
\text { change with respect } \\
\text { to } \mathrm{CCl}_{4} \text { ) }\end{array}$ & $\begin{array}{l}\% \text { Inhibition versus } \mathrm{CCl}_{4} \text { group } \\
\text { (\% increase with respect to VC) }\end{array}$ & $\begin{array}{l}\text { Alleviation (Fold } \\
\text { change with respect } \\
\text { to } \mathrm{CCl}_{4} \text { ) }\end{array}$ \\
\hline $\mathrm{S}-\mathrm{SLNS} \mathrm{s}^{\mathrm{a}}$ & 8 & $52.59 \pm 2.37(1073.93 \pm 166.43)$ & 2.12 & $75.68 \pm 3.61(139.52 \pm 51.44)$ & 4.13 \\
\hline FS & 8 & $34.12 \pm 3.69(1677.97 \pm 298.08)$ & 1.51 & $51.84 \pm 5.88(356.79 \pm 53.86)$ & 2.09 \\
\hline SILY $Y^{a}$ & 25 & $48.84 \pm 3.15(1173.98 \pm 166.43)$ & 1.95 & $75.97 \pm 4.04(129.95 \pm 31.47)$ & 4.18 \\
\hline SR & - & $19.09 \pm 1.38(1677.97 \pm 298.08)$ & 1.23 & $5.22 \pm 2.22(837.98 \pm 148.87)$ & 1.05 \\
\hline
\end{tabular}

Data are expressed as mean \pm S.D $(n=6)$ except for fold change. ${ }^{a}$ All the groups are significantly different from each other at $p \leq 0.001$ except for those marked similarly. $\mathrm{CCl}_{4}$ increased the ALT and AST levels by $2570.07 \%$ and $890.89 \%$ respectively with respect to VC. Actual values for CCl 4 and VC for ALT are $7.21 \pm 0.35$ and $0.27 \pm 0.05$ respectively; AST are $6.50 \pm 0.61$ and $0.93 \pm 0.20$ respectively. The actual values are expressed in units of nmoles/nL. 


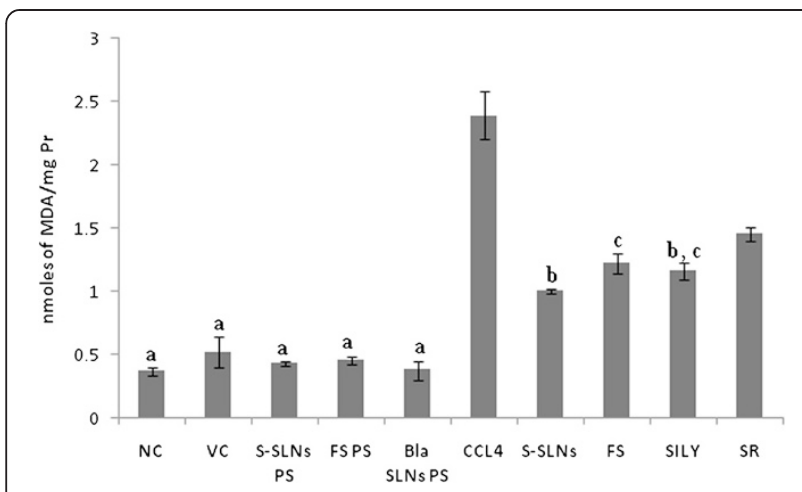

Figure $3 \mathrm{MDA}$ levels after treatment with S-SLNs, FS, SILY and various other control groups post $\mathrm{CCl}_{4}$ induced hepatic injury. Data are expressed as mean \pm S.D $(n=6)$; except NC and PS groups $(n=3)$. All groups are significantly different from each other at $p<0.001$ or $p<$ 0.050 except for those marked similarly.

significant usefulness of phytochemical loaded SLNs over free drugs is available on the utility of SLNs for liver disorders.

S-SLNs with total drug content of $3.31 \pm 0.01 \mathrm{mg} / \mathrm{ml}$ and \% entrapment of $73.92 \pm 2.49 \%(\mathrm{n}=3)$, with an average particle size and zeta potential of $120.30 \mathrm{~nm}$ (PDI0.111 ) and $-51.31 \mathrm{mV}$, respectively, were prepared by the microemulsification method. The method of preparation and the formula have been optimized in our laboratory $[18,33]$. It may also be highlighted here that the method of preparation and the formula optimized in our laboratory [28,33] uses dilution of hot microemulsion with a significantly small (1:1) quantity of cold water (usual recommended volume is $1: 25$ to $1: 100$ ) such that it results in a concentrated dispersion. Latter ensures (i) a higher drug content/volume of the dispersion such that suitable doses can be administered in smaller volumes and, (ii) overcomes the need to concentrate the

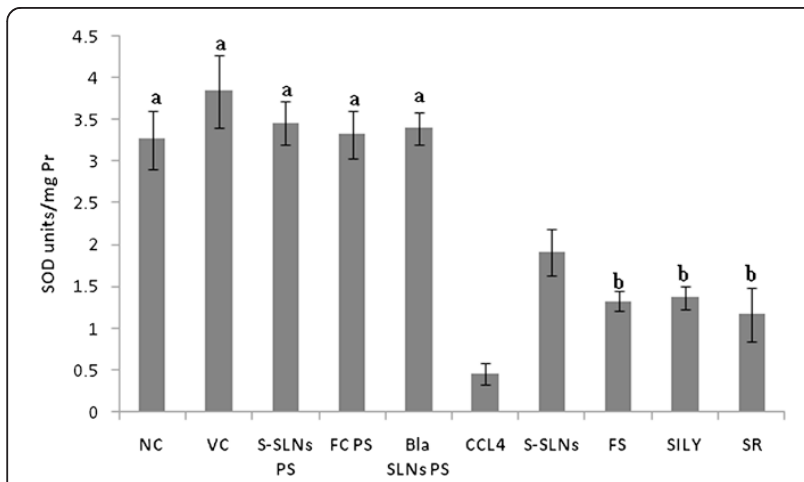

Figure 4 SOD levels following treatment with S-SLNs, FS, SILY and various other control groups post $\mathrm{CCl}_{4}$ induced hepatic injury. Data are expressed as mean \pm S.D $(n=6)$; except NC and PS groups $(n=3)$. All groups are significantly different from each other at $p<0.001$ or $p<0.050$ except for those marked similarly.

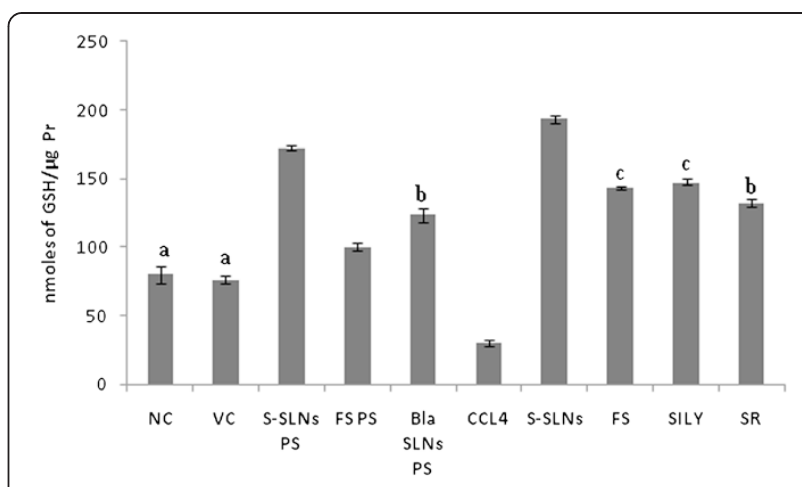

Figure 5 GSH levels after treatment with S-SLNs, FS, SILY and various other control groups post $\mathrm{CCl}_{4}$ induced hepatic injury. Data are expressed as mean \pm S.D $(n=6)$; except NC and PS groups $(n=3)$. All groups are significantly different from each other at $p<0.001$ or $p<0.050$ except for those marked similarly.

dispersion by dialysis or lyophilisation. Both of which are time consuming and costly techniques.

Size of nanoparticles monitors their uptake, into pathological and inflamed tissues by macrophages, or delivery across the fenestrae of the liver sinusoid $[41,42]$. It has been reported that nanoparticles with a diameter of less than $200 \mathrm{~nm}$ reach liver parenchymal cells and generate significant effects $[43,44]$. Presence of tween 80 as a surfactant in these SLNs gives them an added advantage of hydrophilicity similar to that imparted by PEGylation $[27,30,44]$. Nanoparticles having zeta potential $> \pm 25 \mathrm{mV}$ are stable dispersions with little or no chance of aggregation. Furthermore, a PDI of $<0.3$ confirms uniform distribution of nanosized particles in the SLN dispersion with low or no incidence of micrometer particles. The latter may also indirectly be taken to confirm that all particles are of nano size and there are no aggregates. The small size of the stable and uniform SSLN dispersion is expected to result in cumulative uptake by the pathological liver over prolonged period due

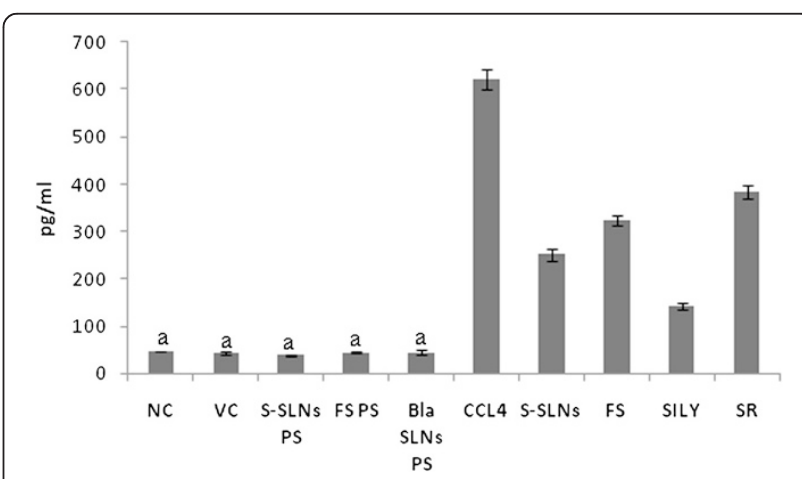

Figure 6 TNF-a levels after treatment with S-SLNs, FS, SILY and various other control groups post $\mathrm{CCl}_{4}$ induced hepatic injury. Data are expressed as mean \pm S.D $(n=6)$; except NC and PS groups $(n=3)$. All groups are significantly different from each other at $p<0.001$ except for those marked similarly. 
to repetitive filtration and passes through a probable facilitated transport across the fenestrae of the liver sinusoids. He et al. [40] have reported 2.79 times improved oral BA of SILY upon incorporation into SLNs (170.7 nm) which could result in a better drug targeting to the liver.

Previously we have reported that drug released versus time data for S-SLNs wherein the total amount of the drug released upto $24 \mathrm{~h}$ is $69.8 \%$ while a fast release of $28.4 \%$ in initial $1 \mathrm{~h}$ indicated the burst release of the drug either from the small sized nanoparticles or the release of the unentrapped drug [27]. Further, a controlled release pattern was depicted during 6-11 h, where no significant increase in the \% drug released was observed [27]. In the oral pharmacokinetics conducted by us (Figure 7), in a separate study in mice, at a dose of 4 $\mathrm{mg} / \mathrm{kg} \mathrm{BW}$, administered S-SLNs indicated a 1.32 times better $(\mathrm{p}<0.05) \mathrm{BA}\left(\mathrm{AUC}_{0-\infty}\right)$ than FS (unpublished work). It may however be noted that the study was performed in mice and at a lower dose of $4 \mathrm{mg} / \mathrm{kg} \mathrm{BW}(8$ $\mathrm{mg} / \mathrm{kg}$ BW dose of sesamol was administered to rats presently) and since the latter are usually fast metabolisers so the effect achieved with S-SLNs in rats and also in humans, may actually be more pronounced. Further, a higher $t_{\max }(8 \mathrm{~h})$ value observed for S-SLNs, in comparison to $1 \mathrm{~h}$ for FS, indicates a sustained effect, probably achieved due to slow release of the drug from the lipidic nanoparticles (unpublished work).

Presently, $\mathrm{CCl}_{4}$ administration lead to hepatic steatosis, inflammation, and the formation of the vascular and fibrotic bridges between nodules as revealed by the histological studies and these observations were similar to previous reported studies $[27,35]$. Present investigations revealed improvement in histoarchitecture of liver on treatment with S-SLNs which was significantly better than FS and SR groups and comparable to the SILY group. In an earlier report sesamol exhibited renoprotective effects in streptozotocin (STZ) induced diabetes in

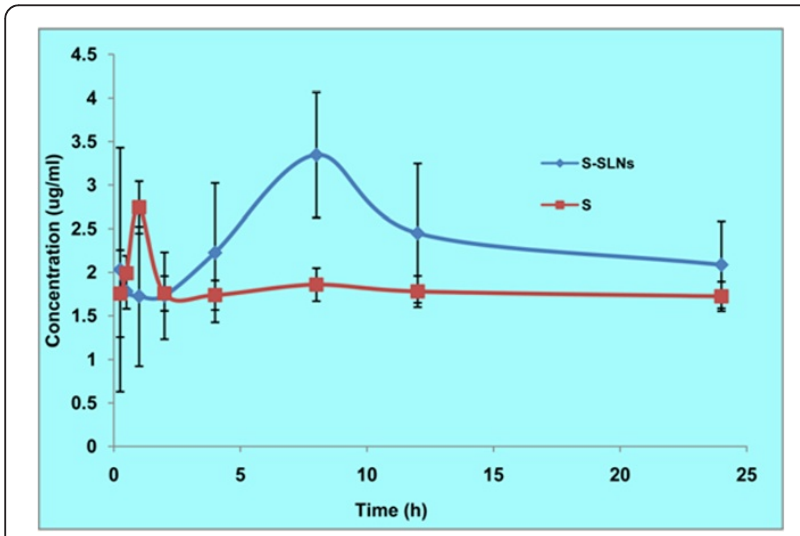

Figure 7 Plasma concentration-time profiles of S-SLNs and FS when administered orally at a dose of $4 \mathrm{mg} / \mathrm{kg}$ to mice.* $\mathrm{S}$ here stands for free sesamol i.e. FS. rats [45] as it modulated the release of profibrotic cytokines i.e. tissue growth factor- 1 beta (TGF- $\beta 1$ ). Similar effect in the present study may have led to reduction in the fibrotic bridges in S-SLNs treated group and better effects observed in comparison with FS and SR groups.

Administration of $\mathrm{CCl}_{4}$ to rats lead to a significant elevation $(\mathrm{p}<0.001)$ in the levels of serum liver injury markers, i.e. ALT and AST in comparison with the untreated VC and NC group. Increase in AST and ALT levels is attributed to the damaged structural integrity of the liver, as these enzymes are cytoplasmic in location and hence are released into the plasma due to the liver cell damage [46]. Treatment with S-SLNs significantly reduced the serum ALT and AST levels, which were significantly better than FS group and was comparable to SILY (as shown in Table 2), though the latter was administered at a 3 times higher dose of $25 \mathrm{mg} / \mathrm{kg}$ BW. Sesamol has exhibited anti-MMP-9 (matrix metallopeptidase 9) activity against monocrotaline-induced SOS in rats [14]. MMP-9 is important for the breakdown and necrosis of hepatocytes [47] resulting in the leakage of ALT and AST from liver. Hence, it may indirectly indicate that the antiMMP-9 effect of sesamol [23] may protect against hepatic tissue necrosis, by attenuating the increased levels of ALT and AST.

S-SLNs treatment may lead to membrane stabilization of hepatocytes which is indicated presently by the reduction of LPO. $\mathrm{CCl}_{4}$ hepatotoxicity is attributed to $\mathrm{LPO}$ and increased MDA levels [48] in liver tissue. Administration of S-SLNs significantly reduced $(\mathrm{p}<0.001)$ the $\mathrm{CCl}_{4}$ induced increase in MDA levels and the effect was better $(\mathrm{p}<0.05)$ than FS group. This may be assigned to the higher cellular permeability of sesamol achieved by S- SLNs [49]. S-SLNs with a polysorbate 80 and a phospholipid coat of surfactant and co-surfactant respectively, are expected to intermingle with the membrane lipids of liver cells resulting in physiologically significant effects as shown in the present study.

A decreased SOD activity observed in the $\mathrm{CCl}_{4}$ treated group was attributable to its easy inactivation by lipid peroxides or ROS. SOD is a sensitive index for the hepatocellular damage [50]. Since treatment with S-SLNs significantly lowered the levels of MDA or ROS, hence, as expected SOD was significantly $(\mathrm{p}<0.05)$ upregulated in S-SLNs group. It is important to note here that S-SLNs treatment was not only significantly more effective than FS but also in comparison to a well established hepatoprotective and antioxidant SILY, presently in clinical use too [51].

GSH is a predominant low-molecular-weight thiol and the most important nonenzyme antioxidant in mammalian cells [52]. It effectively protects cells against oxidative stress caused damage by scavenging free radicals, removing hydrogen peroxide $\left(\mathrm{H}_{2} \mathrm{O}_{2}\right)$, and suppressing LPO [53]. In our study, treatment with S-SLNs increased 
the levels of GSH significantly ( $\mathrm{p}<0.001)$ as compared to all other groups. Further, even the S-SLNs PS and Bla SLNs PS groups showed an increase in the levels of GSH as compared to $\mathrm{NC}$ and $\mathrm{VC}$ groups. These results indicate that the SLNs have a unique ability to increase the levels of GSH in vivo. These results are similar to those observed and reported by us previously [18,27]. Our formulation contains soy lecithin (a type of phospholipid component of biological cell membranes) which consists of $21 \%$ posphatidylcholine, and was administered daily for a time period of four weeks, and could probably lead to an increase in the intrinsic levels of GSH in hepatic tissue. Intake of phosphatidylcholine for a long duration is reported to result in an increase in glutathione levels [54]. Even though the \% of soya lecithin in our formulation is very small (0.58\%) but since it is now incorporated into a highly bioavailable form, i.e. SLNs, the effect may be observed even at low concentrations.

Hepatic injury is associated with elevated TNF- $\alpha$ level as observed presently in the $\mathrm{CCl}_{4}$ group and also reported elsewhere [55]. TNF- $\alpha$ levels are elevated both in the infiltrating inflammatory cells and hepatocytes in chronic liver injuries, including viral or alcoholic liver diseases, hepatitis, ischemia, and biliary obstruction [56]. Exposure to hepatotoxic chemicals facilitates TNF- $\alpha$ to induce necrotic cell death and hepatic apoptosis [57]. Sesamol attenuated TNF- $\alpha$ levels in STZ induced diabetes in rats [45] and in our study also elevated levels of TNF- $\alpha$ were significantly attenuated $(\mathrm{p}<0.001)$ by S-SLN treatment and the effect was significantly $(\mathrm{p}<0.001)$ better than FS group.

\section{Conclusion}

S-SLNs treatment was not only significantly more effective than FS but, also showed effects comparable to a wellestablished hepatoprotective and antioxidant agent SILY. It may however be noted here that the recommended and presently used dose of SILY $(25 \mathrm{mg} / \mathrm{kg})$ is $>3$ times that used for S-SLNs $(8 \mathrm{mg} / \mathrm{kg})$. Hence it may be indirectly concluded that S-SLNs are a significantly better therapeutic than SILY. Significant research is now focused on establishing the multiple biological roles of antioxidant phytochemicals. However, in most of these cases, the effects remain confined to the in vitro laboratory experiments due to lack of translation of their antioxidant activity to physiological systems. Major limitations to the latter are poor solubility, stability (proneness to oxidative and photodegradation), permeability, and bioavailability coupled with a fast metabolism of these molecules. Furthermore the in vitro or preclinical doses at which the effects are obtained are not translatable to human use. Hence their biopharmaceutical enhancement is a must if they have to 'see the light' of being clinically relevant. Although solubility and BA of sesamol are significant, however its fast metabolism and subsequent elimination indicates need to package it into a sustained/ prolonged release carrier system to improve its $\mathrm{T}_{1 / 2}$ and hence mean residence time in the body to elicit considerable physiological effect. It may be noted that earlier reported data uses higher doses and parenteral administration of sesamol. Presently an orally administered formulation of sesamol is reported. Furthermore the Bla-SLNs PS groups confirm the absence of any adverse effects and safety of the developed formulation. The study established that sesamol could be used as a therapeutic agent for the treatment of liver diseases provided it is loaded into a suitable delivery system.

\section{Abbreviations \\ ALT: Alanine aminotransferase; AST: Aspartate aminotransferase: \\ BA: Bioavailability; BW: Body weight; Bla-SLNs: Blank-solid lipid nanoparticles; CCI4: Carbon tetrachloride; DTNB: 5-5' dithiobis-2-2 nitrobenzoic acid; FS: Free sesamol; GSH: Reduced glutathione; HE: Haemotoxylin and eosin; LPO: Lipid peroxidation; MDA: Malondialdehyde; NC: Naïve control; PMS: Post -mitochondrial supernatant; PS: Per se; PDI: Polydispersity index; ROS: Reactive oxygen species; S-SLNs: Sesamol loaded solid lipid nanoparticles; SR: Self recovery; SLNs: Solid lipid nanoparticles; SOD: Superoxide dismutase; SILY: Silymarin; TDC: Total drug content; VC: Vehicle control.}

\section{Competing interests}

The authors declare that they have no competing interests.

\section{Authors' contributions}

NS has made contribution in conducting all experiments, acquisition of data, analysis and drafting of the manuscript. NK has made contribution in conception and design of the experiment. VK has made contribution in carrying out experiments related to preparation and characterization of the solid lipid nanoparticles. IPK has contributed in conception and design of the experiment and interpretation of data, drafting and revising the manuscript for intellectual content. All authors read and approved the final manuscript.

\section{Acknowledgements}

The authors are thankful to Sophisticated Analytical Instrumentation Facility (SAIF), Panjab University, Chandigarh for TEM analysis.

\section{Author details}

1Department of Biotechnology, Panjab University, Chandigarh 160014, India. ${ }^{2}$ Department of Pharmaceutics, University Institute of Pharmaceutical Sciences, Panjab University, Chandigarh 160014, India.

Received: 17 July 2014 Accepted: 17 April 2015

Published online: 03 May 2015

\section{References}

1. Rivera-Espinoza Y, Muriel P. Pharmacological actions of curcumin in liver diseases or damage. Liver Int. 2009;29:1457-66.

2. Muriel P, Rivera-Espinoza Y. Beneficial drugs for liver diseases. J Appl Toxicol. 2008:28:93-103.

3. Morisco F, Vitaglione P, Amoruso D, Russo B, Fogliano V, Caporaso N. 144-150: foods and liver health. Mol Aspects Med. 2008;29:144-50.

4. Rechnagel RO, Glende EAJ. Carbon tetrachloride hepatotoxicity: an example of lethal cleavage. CRC Crit Rev Toxicol. 1973;2:263-97.

5. Slater TF. Free-radical mechanisms in tissue injury. Biochem J. 1984;222:1-15.

6. Liu SL, Degli-Esposti S, Yao T, Diehl AMA, Zern MA. Vitamin E therapy of acute CCl4-induced hepatic injury in mice is associated with inhibition of nuclear factor kappa $\beta$ binding. Hepatology. 1995;22:1474-81.

7. Hernandez-Munoz R, Diaz-Munoz M, Chagoya de Sanchez V. Possible role of cell redox state on collagen metabolism in carbon tetrachloride-induced cirrhosis as evidenced by adenosine administration to rats. Biochim Biophys Acta. 1994;1200:93-9. 
8. Weber LW, Boll MA, Stampfl A. Hepatotoxicity and mechanism of action of haloalkanes: carbon tetrachloride as a toxicological model. Crit Rev Toxicol. 2003;33:105-39.

9. Elleuch M, Besbes S, Roiseux O, Blecker C, Attia H. Quality. Characteristics of sesame seeds and by- products. Food Chem. 2007;103:641-50.

10. Mohamed HMA, Awatif II. The Use of sesame Oil Unsaponifiable. Matter as a natural antioxidant. Food Chem. 1998;62:269-76.

11. Hemalatha G, Pugalendi KV, Saravanan R. Modulatory effect of sesamol on DOCA-salt-induced oxidative stress in uninephrectomized hypertensive rats. Mol Cell Biochem. 2013;379:255-65.

12. Suja KP, Jayalekshmy A, Arumughan C. Free radical scavenging behavior of antioxidant compounds of sesame (Sesamum indicum L.) in DPPH $\left(^{*}\right)$ system. J Agric Food Chem. 2004;52:912-5.

13. Chennuru A, Saleem MTS. Antioxidant, Lipid Lowering, and Membrane Stabilization Effect of Sesamol against Doxorubicin-Induced Cardiomyopathy in Experimental Rats. BioMed Res Int. 2013;2013:934239. doi:10.1155/2013/934239.

14. Kumagai $Y$, Lin LY, Schmitz DA, Cho AK. Hydroxyl radical mediated demethylenation of (methylenedioxy) phenyl compounds. Chem Res Toxicol. 1991:4:330-4.

15. Jan KC, Ho CT, Hwang LS. Bioavailability and tissue distribution of sesamol in rat. J AgricFood Chem. 2008;56:7032-7.

16. Jan KC, Ho CT, Hwang LS. Elimination and metabolism of sesamol, a bioactive compound in sesame oil, in rats. Mol Nutr Food Res. 2009:53:\$36-43.

17. CBBD: http://toxnet.nlm.nih.gov/cpdb/chempages/SESAMOL.html. 2007.

18. Kakkar V, Mishra M, Chuttani K, Chopra K, Kaur IP. Delivery of sesamol loaded solid lipid nanoparticles to the brain for menopause related emotional and cognitive central nervous system derangements. Rejuvenation Res. 2011;14:597-604.

19. Hsu DZ, Chen KT, Li YH, Chuang YC, Liu MY. Sesamol delays mortality and attenuates hepatic injury after cecal ligation and puncture in rats: role of oxidative stress. Shock. 2006;25:528-32.

20. Hsu DZ, Chien SP, Chen KT, Liu MY. The effect of sesamol on systemic oxidative stress and hepatic dysfunction in acutely iron-intoxicated mice. Shock. 2007;28:596-601.

21. Chandrasekaran VRM, Chien SP, Hsu DZ, Liu MY. Anti-hepatotoxic effects of 3,4-methylenedioxyphenol and $\mathrm{N}$-acetylcysteine in acutely acetaminophenoverdosed mice. Hum Exp Toxicol. 2011;30:1609-15.

22. Chandrasekaran VR, Hsu DZ, Liu MY. The protective effect of sesamo against mitochondrial oxidative stress and hepatic injury in acetaminophenoverdosed rats. Shock. 2009:32:89-93.

23. Periasamy S, Hsu DZ, Chen SY, Yang SS, Chandrasekaran VR, Liu MY. Therapeutic sesamol attenuates monocrotaline-induced sinusoidal obstruction syndrome in rats by inhibiting matrix metalloproteinase-9. Cell Biochem Biophys. 2011;61:327-36.

24. Inaneshwari S, Hemshekhar M, Thushara RM, Sundaram MS, Santhosh MS, Sunitha K, et al. Sesamol Ameliorates Cyclophosphamide-Induced Hepatotoxicity by Modulating Oxidative Stress and Inflammatory Mediators. Anticancer Agents Med Chem. 2014;14:975-83.

25. Ohta S, Suzuki M, Sato N, Kamogawa A, Shinoda M. Protective effects of sesamol and its related compounds on carbon tetrachloride induced liver injury in rats. Yakugaku Zasshi. 1994;114:901-10.

26. Uchida M, Nakajin S, Toyoshima S, Shinoda M. Antioxidative effect of sesamol and related compounds on lipid peroxidation. Biol Pharm Bull. 1996;19:623-6

27. Singh N, Khullar N, Kakkar V, Kaur I P: Hepatoprotective effects of sesamo loaded solid lipid nanoparticles in carbon tetrachloride induced sub-chronic hepatotoxicity in rats. Environ Toxicol. 2014; doi:10.1002/tox.22064.

28. Kakkar V, Singh S, Singla D, Kaur IP. Exploring solid lipid nanoparticles to enhance the oral bioavailability of curcumin. Mol Nutr Food Res. 2011:55:495-503.

29. Bhandari R, Kaur IP. Pharmacokinetics, tissue distribution and relative bioavailability of isoniazid-solid lipid nanoparticles. Int J Pharm. 2013:441:202-12.

30. Singh N, Khullar N, Kakkar V, Kaur IP. Attenuation of Carbon TetrachlorideInduced Hepatic Injurywith Curcumin-Loaded Solid Lipid Nanoparticles. BioDrugs. 2014;28:297-312

31. Pradhan SC, Girish C. Hepatoprotective herbal drug, silymarin from experimental pharmacology to clinical medicine. Indian J Med Res. 2006:124:491-504.

32. Manjunath K, Reddy S, Venkateswarlu V. Solid lipid nanoparticles as drug delivery systems. Method Exp Clin. 2005;27:127-44.
33. Kakkar V, Kaur IP. Preparation, characterization and scale-up of sesamol loaded solid lipid nanoparticles. Nanotechnol Dev. 2012;2, e8.

34. Gopal N, Sengottuvelu S. Hepatoprotective activity of Clerodendrum inerme against CCl4 induced hepatic injury in rats. Fitoterapia. 2008;79:24-6.

35. Dong MX, Jia Y, Zhang YB, Li CC, Geng YT, Zhou L, et al. Emodin protects rat liver from $\mathrm{CCl} 4$-induced fibrogenesis via inhibition of hepatic stellate cells activation. World J Gastroenterol. 2009;15:4753-62.

36. Reitman S, Frankel SA. A colorimetric method for the determination of serum glutamic oxalacetic and glutamic pyruvic transaminases. Amer J Clin Pathol. 1957;28:56-63.

37. Wills ED. Mechanisms of lipid peroxide formation in animal tissue. Biochem J. 1966;99:667-76.

38. Kono Y. Generation of superoxide radical during autooxidation of hydroxylamine and an assay for superoxide dismutase. Arch Biochem Biophys. 1978;186:189-95.

39. Jollow D, Mitchell L, Zampaglione N, Gillete J. Bromobenzene induced liver necrosis: protective role of glutathione and evidence for 3,4-bromobenzenoxide as the hepatotoxic intermediate. Pharmacology. 1974;11:151-69.

40. He J, Hou S, Lu W, Zhu L, Feng J. Preparation, Pharmacokinetics and Body Distribution of Silymarin-Loaded Solid Lipid Nanoparticles After Oral Administration. J Biomed Nanotechnol. 2007;3:195-202.

41. Hu Y, Xie J, Tong YW, Wang CH. Effect of PEG conformation and particle size on the cellular uptake efficiency of nanoparticles with the HepG2 cells. J Control Release. 2007:118:7-17.

42. Liang HF, Yang TF, Huang $C T$, Chen MC, Sung HW. Preparation of nanoparticles composed of poly(gamma-glutamic acid)-poly (lactide) block copolymers and evaluation of their uptake by HepG2 cells. J Control Release. 2005:105:213-25.

43. Hashida M, Takemura S, Nishikawa M, Takakura Y. Targeted delivery of plasmid DNA complexed with galactosylated poly(L-lysine). J Control Release. 1998:53:301-10.

44. Li L, Wang H, Ong ZY, Xu K, Ee PLR, Zheng S, et al. Polymer- and lipid-based nanoparticle therapeutics for the treatment of liver diseases. Nano Today. 2010;5:296-312.

45. Kuhad A, Sachdeva AK, Chopra K. Attenuation of renoinflammatory cascade in experimental model of diabetic nephropathy by sesamol. J Agric Food Chem. 2009:57:6123-8

46. Sallie R, Tredger JM, Williams R. Drugs and the liver part 1: testing liver function. Biopharm Drug Dispos. 1991;12:251-9.

47. Ohashi N, Hori T, Chen F, Jermanus S, Eckman CB, Nakao A, et al. Matrix metalloproteinase-9 contributes to parenchymal hemorrhage and necrosis in the remnant liver after extended hepatectomy in mice. World J Gastroenterol. 2012;18:2320-33.

48. Curtis JJ, Mortiz M. Serum enzymes derived from liver cell fraction and response to carbon tetrachloride intoxication in rats. Gastroenterol. 1972;62:84-92.

49. Geetha T, Bhandari R, Kaur IP. Sesamol: An efficient antioxidant with potential therapeutic benefits. Med Chem. 2009;5:367-71.

50. Feher J, Lengyel G. Silymarin in the Prevention and Treatment of Live Diseases and Primary Liver Cancer. Curr Pharm Biotechnol. 2012;13:210-7.

51. Javed S, Kohli K, Ali M. Reassessing bioavailability of silymarin. Altern Med Rev. 2011;16:239-49.

52. Wu G, Fang YZ, Yang S, Lupton JR, Turner ND. Glutathione metabolism and its implications for health. J Nutr. 2004:134:489-92.

53. Blair IA. Endogenous glutathione adducts. Curr Drug Metab. 2006;7:853-72

54. Shukitt-Hale B, Erat SA, Joseph JA. Spatial learning and memory deficits induced by dopamine administration with decreased glutathione. Free Radic Biol Med. 1998:24:1149-58.

55. Roy S, Sannigrahi S, Majumdar S, Ghosh B, Sarkar B. Resveratrol regulates antioxidant status, inhibits cytokine expression and restricts apoptosis in carbon tetrachloride induced Rat hepatic injury. Oxid Med Cell Longev. 2011;703676:12.

56. Hernandez-Munoz I, Torre PDL, Sanchez-Alcazar JA, Garcia I, Santiago E, Munoz-Yague MT, et al. Tumor necrosis factor ainhibits collagen a1(I) gene expression in rat hepatic stellate cells through a G protein. Gastroenterol. 1997; 113:625-40

57. Berghe TV, Denecker G, Brouckaert G, Vadimovisch D, D'Herde KK, Vandenabeele P. More than one way to die: methods to determine TNF-induced apoptosis and necrosis. Methods Mol Med. 2004:98:101-26. 